\title{
SCHUR PRODUCTS OF OPERATORS AND THE ESSENTIAL NUMERICAL RANGE
}

BY

\author{
QUENTIN F. STOUT
}

\begin{abstract}
Let $\mathcal{E}=\left\{e_{n}\right\}_{n=1}^{\infty}$ be an orthonormal basis for a Hilbert space $\mathcal{H}$. For operators $A$ and $B$ having matrices $\left(a_{i j}\right)_{i, j=1}^{\infty}$ and $\left(b_{i j}\right)_{i, j=1}^{\infty}$, their Schur product is defined to be $\left(a_{i j} b_{i j}\right)_{i, j-1}^{\infty}$. This gives $\mathscr{B}(\mathcal{H})$ a new Banach algebra structure, denoted $\mathfrak{B}_{\mathscr{G}}$. For any operator $T$ it is shown that $T$ is in the kernel (hull(compact operators)) in some $\mathscr{B}_{\S}$ iff 0 is in the essential numerical range of $T$. These conditions are also equivalent to the property that there is a basis such that Schur multiplication by $T$ is a compact operator mapping Schatten classes into smaller Schatten classes. Thus we provide new results linking $\mathscr{B}(\mathscr{H}), \mathscr{B}_{\mathbb{S}}$ and $\mathscr{B}(\mathscr{B}(\mathcal{K})$ )).
\end{abstract}

Let $\mathcal{H}$ be a separable infinite-dimensional Hilbert space, and $A, B$ be bounded operators on $\mathcal{H}$. For an orthonormal basis $\mathcal{E}=\left\{e_{n}: 1 \leqslant n<\infty\right\}$ of $\mathcal{H}, A$ and $B$ have matrix representations $\left(a_{i j}\right)_{i, j=1}^{\infty}$ and $\left(b_{i j}\right)_{i, j=1}^{\infty}$. In 1911 Schur $[15$, p. 8] proved that the termwise product $\left(a_{i j} b_{i j}\right)_{i, j=1}^{\infty}$, denoted $A * B$, is itself a bounded operator with norm satisfying $\|A * B\| \leqslant\|A\| \cdot\|B\|$. This multiplication will herein be called Schur multiplication, as opposed to the more common, but falsely crediting, "Hadamard multiplication". Schur's result shows that once a basis $\mathcal{E}$ is selected, then * defines a commutative Banach algebra, denoted by $\mathscr{B}_{\mathscr{E}}$. While there is a surge of interest in applications of Schur multiplication (see, for example, Bennett [3], Johnson and Williams [6], Pommerenke [11], or Styan [18]), litle is known about the structure of $\mathscr{B}_{\mathscr{E}}$. In particular, few connections between the structure of $\mathscr{B}(\mathcal{C})$ and $\mathscr{B}_{\mathscr{G}}$ have been explored, though Varopoulos [20] has asked whether the linear structure of $\mathscr{B}(\mathcal{H})$ forces $\mathscr{B}_{\mathcal{E}}$ to be a $Q$-algebra. Except for Bennett [4] and Ruckle [13], the systematic study of Schur multiplication as a means of obtaining unusual operators in $\mathscr{B}(\mathscr{B}(\mathcal{H}))$ is a relatively untouched concept, even though several interesting operators are Schur multipliers, e.g., Kwapien and Pelczynski [8].

It is important to keep in mind that $A * B$ depends upon a choice of basis. The symbol $A_{\mathscr{G}}$ will be used to denote the element of $\mathscr{B}\left(\mathscr{B}(\mathcal{C})\right.$ ) defined by $A_{\mathscr{G}}(B)=$ $A * B$, where * is with respect to the basis $\mathcal{E}$.

It will be shown that the following conditions (and several others) are equivalent for any operator $T$.

(i) 0 is in the essential numerical range of $T$.

(ii) There exists a basis $\mathcal{E}$ such that $T$ is in $\operatorname{kernel}\left(\right.$ hull $\left(\mathcal{K}_{\mathscr{E}}\right)$ ), where $\mathcal{K}_{\mathscr{E}}$ is the ideal of compact operators in $\mathfrak{B}_{\mathcal{E}}$.

Received by the editors October 19, 1979 and, in revised form, March 3, 1980.

1980 Mathematics Subject Classification. Primary 47A12, 47D99; Secondary 46J99.

Key words and phrases. Schur multiplication, Hadamard multiplication, essential numerical range, matrix representations. 
(iii) There exists a sequence of bases $\mathcal{E}_{n}$ such that $T_{\mathscr{E}_{n}} \rightarrow 0$ uniformly in $\mathscr{B}(\mathscr{B}(\mathcal{H}))$.

(iv) There exists a basis $\mathscr{F}$ such that, for any $p$ with $0<p<\infty$ and any $q$ with $p /(p+1)<q \leqslant \infty, T_{\mathscr{F}}$ is a compact operator mapping the Schatten class $\bigodot_{p}$ into $e_{q}$.

As a motivation for interest in the last condition, notice that for an operator $T$ with 0 in its essential numerical range (for example, any projection with infinite kernel), there is a basis $\mathscr{F}$ such that $T_{\mathscr{F}}$ maps $\mathscr{B}(\mathscr{H})$ into $\mathcal{C}_{2}$, the Hilbert-Schmidt class. Since an operator is in $C_{2}$ if and only if the entries of its matrix with respect to any basis are square summable, this shows a plethora of operators whose entries are not in $l_{2}$, and yet which, when acting as Schur multipliers, map $\mathscr{B}(\mathcal{H})$ into matrices whose entries are in $l_{2}$. Such behavior is impossible with the usual multiplication in $\mathscr{B}(\mathcal{H})$.

This paper is part of the author's Ph.D. thesis written at Indiana University under the supervision of Professor John B. Conway. Mention should also be made of Professor James P. Williams, who motivated the author's original interest in $\mathscr{B}_{\mathscr{E}}$, and of Professor Grahame Bennett, with whom the author has had many discussions.

1. Preliminaries. Let $X$ and $Y$ denote Banach spaces and let $\mathscr{B}(X, Y)$ and $\mathcal{K}(X, Y)$ denote, respectively, the continuous and compact linear operators from $X$ to $Y$. Let $T$ be an operator in $\mathscr{B}(X, Y)$. For any integer $n>0$, the nth approximation number of $T$, denoted $a_{n}(T)$, is given by $a_{n}(T)=\inf \{\|T-F\|: F \in$ $\Re(X, Y), \operatorname{rank}(F) \leqslant n\}$. For $0<p<\infty$ the Schatten $p$ class of operators in $\mathscr{B}(X, Y)$, denoted $\bigodot_{p}(X, Y)$, is here defined to be the set of operators $T$ such that $\sum_{n=0}^{\infty} a_{n}(T)^{p}<\infty$. $\mathcal{C}_{\infty}(X, Y)$ can be taken to be either $\mathscr{B}(X, Y)$ or $\mathcal{K}(X, Y)$ in this paper, though the former is rarely used elsewhere. For a Hilbert space $\mathcal{H}$, $e_{p}(\mathcal{H}, \mathcal{H})$ will be shortened to $e_{p}$.

A quasinorm \|\|$_{p}$ can be defined on $C_{p}$ by $\|T\|_{p}=\left(\sum_{n=0}^{\infty} a_{n}(T)^{p}\right)^{1 / p}$. If $1 \leqslant p \leqslant \infty$, then $e_{p}$ is a Banach space, but for $p<1$, \|\|$_{p}$ is not a true norm. It is complete (see McCarthy, [9, Corollary 3.2]), and there is a constant $c_{p}$ such that $\|A+B\|_{p} \leqslant c_{p}\left(\|A\|_{p}+\|B\|_{p}\right)$. (From [9, Theorem 2.8] one can show $c_{p}=2^{1 / p-1}$ is the best possible value.) Similarly $\Re\left(\bigodot_{p}, \bigodot_{q}\right)$ and $\mathcal{K}\left(\bigodot_{p}, \bigodot_{q}\right)$ will not be Banach spaces if $q \leqslant 1$, but will be complete and satisfy an altered triangle inequality. \|\|$_{p q}$ will be used to denote the quasinorm in these spaces.

Given any $A$ in $\mathscr{B}(\mathcal{H})$ and any basis $\mathcal{E}$, a matrix representation $\left(a_{i j}\right)$ of $A$ can also be viewed as a bounded, continuous function on $\mathbf{N} \times \mathbf{N}$, where $\mathbf{N}$ denotes the natural numbers. Thus $\mathscr{B}_{\mathscr{E}}$ can be imbedded as a subset of $C(\mathbf{N} \times \mathbf{N})$, which is in turn naturally isometrically isomorphic to $C(\beta(\mathbf{N} \times \mathbf{N}))$, where for any completely regular space $X, C(X)$ denotes the Banach algebra of bounded continuous complex-valued functions on $X$, and $\beta(X)$ denotes the Stone-Čech compactification of $X$. These injections allow one to construct the maximal ideal space of $\mathscr{B}_{\mathscr{E}}$, denoted $\Delta_{\tilde{\delta}}$. (This will make use of the natural identification between maximal closed regular ideals and multiplicative linear functionals.) 
1.1. TheOREM. The map $(i, j) \mapsto\left(A e_{j}, e_{i}\right)$ of $\mathbf{N} \times \mathbf{N}$ into $\Delta_{\mathcal{E}}$ extends to a homeomorphism of $\cup\left\{D^{-}: D \subset \mathbf{N} \times \mathbf{N}\right.$ such that, for all $i$ in $\mathbf{N}$ the cardinalities of $D \cap\{i\}$ $\times \mathbf{N}$ and of $D \cap \mathbf{N} \times\{i\}$ are not greater than one $\}$, as a subset of $\mathscr{B}(\mathbf{N} \times \mathbf{N})$, onto $\Delta_{\mathcal{E}}$ with the regular or hull-kernel topology (i.e., $\mathscr{B}_{\mathfrak{E}}$ is a regular Banach algebra).

A proof of this theorem can be found in Stout [17].

Recall that an element of $\mathscr{B}(\mathscr{H})$ is a compact operator if and only if for any sequence of projections $\left\{P_{n}\right\}$ converging strongly to the identity, $A-P_{n} A P_{n}$ converges to 0 in norm. If $\mathcal{E}=\left\{e_{n}\right\}_{n=1}^{\infty}$ is a basis and $P_{n}$ is taken to be the orthogonal projection onto the first $n$ basis vectors, then it is easy to see that the compact operators form an ideal in $\mathscr{B}_{\mathscr{E}}$. Denoting this ideal by $\mathcal{K}_{\mathscr{E}}$, it is also straightforward that for any $T$ in $\mathscr{B}_{\mathscr{E}}, T$ will be in every maximal ideal which contains $\mathcal{K}_{\mathscr{E}}$ (i.e., $T$ is in kernel(hull $\left.\left(\mathcal{K}_{\mathscr{E}}\right)\right)$ ) if and only if for every $\varepsilon>0, t$ has only finitely many entries with absolute value greater than $\varepsilon$. A matricial property related to this is the small entry property: $T$ in $\mathscr{B}(\mathcal{C})$ has the small entry property if for every $\varepsilon>0$ there is a basis $\left\{e_{n}\right\}$ such that $\left|\left(T e_{n}, e_{n}\right)\right|<\varepsilon$ for all $n$ and $m$. Clearly no nonzero scalar can have either of these properties, nor can any operator close to a scalar.

For an operator $T$ we use $W_{e}(T)$ to denote its essential numerical range, i.e., the numerical range of the image of $T$ in the Calkin algebra $\mathscr{B}(\mathcal{H}) / \mathscr{K}(\mathcal{H})$. For any $T, W_{e}(T)$ is nonempty, compact, and satisfies $W_{e}(T+\lambda)=W_{e}(T)+\lambda$ for any scalar $\lambda$. Further, $W_{e}(T)=\{0\}$ if and only if $T$ is compact. Proofs of these assertions may be found in Bonsall and Duncan [5]. The following theorem states only a few of the conditions known to be equivalent to $0 \in W_{e}(T)$. Proofs may be found in Anderson [1] or Anderson and Stampfli [2].

1.2. ThEOREM. For any $T$ in $\mathscr{B}(\mathcal{H})$ the following are equivalent.

(a) $0 \in W_{e}(T)$.

(b) There exists a sequence $\left\{f_{n}\right\}$ of unit vectors converging weakly to 0 such that $\left(T f_{n}, f_{n}\right) \rightarrow 0$.

(c) There exists a basis $\left\{e_{n}\right\}$ such that $\left(T e_{n}, e_{n}\right) \rightarrow 0$.

(d) $T=A X-X A$ for some $X, A$ in $\mathscr{B}(\mathcal{H})$ with $A$ selfadjoint.

(e) For any $p>1$ and any $\varepsilon>0$ there exists a basis $\left\{e_{n}\right\}$ such that $\Sigma_{n}\left|\left(T e_{n}, e_{n}\right)\right|^{p}$ $<\varepsilon$.

It should be mentioned that Anderson's proof of the equivalence of (a) and (e) uses two deep facts: the equivalence of (a) and (d), and Kuroda's result [7] that for any selfadjoint operator $A$, any $p>1$, and any $\varepsilon>0$, there is a diagonal operator $D$ such that $\|A-D\|_{p}<\varepsilon$. One by-product of the main theorem is a direct proof of this equivalence.

2. The main theorem. The next two lemmas are used repeatedly in the remainder of the paper. 
2.1. Lemma. Suppose $N(n)$ is a sequence of positive integers and $\varepsilon(n)$ is a sequence of positive numbers. If $T \in \mathscr{B}(\mathcal{H})$ and $\left\{e_{n}\right\}$ is a basis such that $\left(T e_{n}, e_{n}\right) \rightarrow 0$, then there is a new basis $\left\{e_{n}^{i}: n \in \mathbf{N}, 1 \leqslant i \leqslant N(n)\right\}$ such that, for all $n$ and $m$ with $n \neq m$, and all $1 \leqslant h \leqslant N(m), 1 \leqslant i, j, k, l \leqslant N(n)$,

(a) $\left|\left(T e_{n}^{i}, e_{m}^{h}\right)\right| \leqslant 2\|T\| / N(\max (n, m))^{1 / 2}$.

(b) $\left|\left(T e_{n}^{i}, e_{n}^{j}\right)\right| \leqslant 2\|T\| / N(n)$.

(c) $\left|\left(T e_{n}^{i}, e_{n}^{j}\right)-\left(T e_{n}^{k}, e_{n}^{l}\right)\right| \leqslant \varepsilon(n)$.

Proof. Define a new sequence $\{\delta(n)\}$ of positive real numbers by $\delta(n)=$ $\varepsilon(n) /\left(2 \max \left\{N(i)^{2}: 1 \leqslant i \leqslant n\right\}\right)$. We first show that $\left\{e_{n}\right\}$ can be relabeled as $\left\{g_{n}^{i}\right.$ : $n=1,2, \ldots, 1 \leqslant i \leqslant N(n)\}$ with $\max \left\{\left|\left(T g_{m}^{i}, g_{n}^{j}\right)\right|,\left|\left(T g_{n}^{j}, g_{m}^{i}\right)\right|\right\}<\delta(n)$ for all $m<$ $n$ and $1 \leqslant i \leqslant N(m), 2 \leqslant j \leqslant N(n)$. To show this, let $g_{1}^{1}=e_{1}$. As $n \rightarrow \infty$ we have $\left(T g_{1}^{1}, e_{n}\right) \rightarrow 0,\left(T e_{n}, g_{1}^{1}\right) \rightarrow 0$ and $\left(T e_{n}, e_{n}\right) \rightarrow 0$, so there exists a $k>1$ such that $\max \left\{\left|\left(T g_{1}^{1}, e_{k}\right)\right|,\left|\left(T e_{k}, g_{1}^{1}\right)\right|, \mid\left(T e_{k}, e_{k}\right)\right\} \leqslant \delta(1)$. Let $g_{1}^{2}=e_{k}$. Since also $\left(T g_{1}^{2}, e_{n}\right) \rightarrow 0$ and $\left(T e_{n}, g_{1}^{2}\right) \rightarrow 0$, we can select an $l>k$ so that setting $g_{1}^{3}=e_{l}$ satisfies $\max \left\{\left|\left(T g_{1}^{i}, g_{1}^{3}\right)\right|,\left|\left(T g_{1}^{3}, g_{i}^{i}\right)\right|\right\} \leqslant \delta(1)$ for all $1 \leqslant i<3$. Continuing in this manner one can find $g_{1}^{i}$ for $i \leqslant N(1)$. Then let $g_{2}^{1}$ be the element of least index among $\left\{e_{n}\right\}$ not already chosen. One can now find $g_{2}^{2}, g_{2}^{3}, \ldots$ as before.

Notice that the only estimate available for $\left(T g_{m}^{1}, g_{n}^{1}\right)$ is $\left|\left(T g_{m}^{1}, g_{m}^{1}\right)\right|<\|T\|$.

For each positive integer $k$ let $\omega_{k}$ denote a primitive $k$ th root of unity, and let $M(k)$ denote the $k \times k$ matrix whose $(i, j)$ entry is $\omega_{k}^{(i-1)(j-1)} / \sqrt{ } k$. Viewing $M(N(n))$ as an operator on the subspace of $\mathcal{H}$ with basis $g_{n}^{1}, \ldots, g_{n}^{N(n)}$, let $e_{n}^{i}=M(N(n))\left(g_{n}^{i}\right)$. Since $M(k)$ is always unitary, $e_{n}^{1}, \ldots, e_{n}^{N(n)}$ forms a new basis for this subspace. Straightforward computations show that (a), (b) and (c) are satisfied.

The following lemma, offered without proof, is probably well known. It is interesting to contrast these bounds with those in Theorem 6.2 of Bennett [4], which gives norms for multipliers on $\mathscr{B}\left(l_{p}(n), l_{q}(n)\right)$. The differences occur because a given matrix has larger multiplier norm on $\Re\left(l_{2}, l_{2}\right)$ than on $C_{2}$.

2.2. Lemma. (a) Let $P_{n}$ be an $n$-dimensional orthogonal projection. Then the map $T \rightarrow P_{n} T P_{n}$ from $e_{p}$ to $\bigodot_{q}$ has norm $n^{\max (0,1 / q-1 / p)}$ for any $p$ and $q$ in $(0, \infty]$.

(b) Let $B=\left(b_{i j}\right)$ be a matrix such that $b_{i j}=0$ if $j>0$ and $\left|b_{i j}\right|<1$ if $j<n$. Then, as multipliers from $\bigodot_{p}$ to $\bigodot_{q}, B$ and its transpose have norms no greater than $n^{\max (0,1 / q-1 / 2,1 / 2-1 / p, 1 / q-1 / p)}$ for all $p$ and $q$ in $(0, \infty]$. Further, given $n$, there is $a$ matrix $B$ so that the multiplier norm of $B$ in $\mathscr{B}\left(\bigodot_{p}, \bigodot_{q}\right)$ attains the bound given for all $p$ and $q$ in $(0, \infty]$.

We now give the main theorem.

2.3. THEOREM. For any $T$ in $\mathscr{B}(\mathcal{H})$ the following conditons are equivalent.

(a) $0 \in W_{e}(T)$.

(b) There is a basis $\mathcal{E}$ such that $T \in \operatorname{kernel}\left(\operatorname{hull}\left(\mathcal{K}_{\mathscr{E}}\right)\right)$.

(c) $T$ has the small entry property.

(d) There exists a sequence of bases $\mathcal{E}(n)$ such that $T_{\mathscr{G}(n)} \rightarrow 0$ uniformly in $\mathscr{B}(\mathscr{B}(\mathscr{H}))$. 
(e) For every sequence $\left\{\alpha_{n}\right\}$ of positive numbers with $\left\{\alpha_{n}\right\} \notin l_{1}$, there is a basis $\left\{e_{n}\right\}$ such that $\left|\left(T e_{n}, e_{n}\right)\right| \leqslant \alpha_{n}$ for all $n$.

(f) There is a basis $\mathcal{E}$ such that for all $q$ in $(0, \infty]$ and $r$ in $(q /(q+1), \infty]$, $T_{\mathcal{E}} \in \mathcal{K}\left(\mathcal{C}_{q}, e_{r}\right)$.

Proof. It is straightforward, using Theorem 1.2, that (e) $\rightarrow$ (a), (d) $\rightarrow$ (c) and (f) $\rightarrow$ (b) $\rightarrow$ (a). Therefore, it will suffice to show (c) $\rightarrow$ (a), (a) $\rightarrow$ (e), (a) $\rightarrow$ (d) and (a) $\rightarrow$ (f).

(c) $\rightarrow$ (a): If $T$ has the small entry property, then for any $\varepsilon>0$ there is a basis so that all entries of the matrix of $T$ have absolute value less than $\varepsilon$. In particular, the diagonal entries of the matrix must have an accumulation point $\lambda$ with $|\lambda| \leqslant \varepsilon$. By Theorem 1.2 and the scalar translation property of numerical ranges, $\lambda \in W_{e}(T)$. Since $W_{e}(T)$ is closed, $0 \in W_{e}(T)$.

(a) $\rightarrow$ (e): Without loss of generality we may assume that $\left\{\alpha_{n}\right\}$ is nonincreasing. Assuming (a) to be true, Theorem 1.2 guarantees the existence of a basis $\left\{f_{n}\right\}$ such that $\left(T f_{n}, f_{n}\right) \rightarrow 0$ as $n \rightarrow \infty$. We construct a new basis $\left\{e_{n}\right\}$ as follows: if $\left|\left(T f_{1}, f_{1}\right)\right|$ $\leqslant \alpha_{1}$, let $e_{1}=f_{1}$. Otherwise let $N$ be as small as possible such that $\sum_{n=1}^{N} \alpha_{n}>$ $\left|\left(T e_{1}, e_{1}\right)\right|$. Pick distinct vectors $f_{2}^{\prime}, \ldots, f_{N}^{\prime}$ from $\left\{f_{n}\right\}$ such that

$$
\left|\left(T f_{i}^{\prime}, f_{i}^{\prime}\right)\right| \leqslant\left(\sum_{n=1}^{N} \alpha_{n}-\left|\left(T f_{1}, f_{1}\right)\right|\right) / N
$$

for $2 \leqslant i \leqslant N$. This will insure that $\left|\left(T f_{1}, f_{1}\right)\right|+\sum_{i=2}^{N}\left|\left(T f_{i}^{\prime}, f_{i}^{\prime}\right)\right|<\sum_{n=1}^{N} \alpha_{n}$.

There is a $\beta_{1}$ in $(0,1)$ such that, if $e_{1}=\beta_{1} f_{1}+\sqrt{1-\beta_{1}^{2}} f_{2}^{\prime}$, then $\left|\left(T e_{1}, e_{1}\right)\right|=\alpha_{1}$. Letting $e_{2}^{\prime}=\sqrt{1-\beta_{1}^{2}} f_{1}-\beta_{1} f_{2}^{\prime}$, we have $e_{1}$ and $e_{2}^{\prime}$ spanning the same subspace as $f_{1}$ and $f_{2}^{\prime}$. Since the trace is invariant on finite-dimensional subspaces we must have $\left|\left(T e_{2}^{\prime}, e_{2}^{\prime}\right)\right|+\sum_{i=3}^{N}\left|\left(T f_{i}^{\prime}, f_{i}^{\prime}\right)\right| \leqslant \sum_{n=2}^{N} \alpha_{n}$. If $N=2$, or if it should otherwise happen that $\left|\left(T e_{2}^{\prime}, e_{2}^{\prime}\right)\right| \leqslant \alpha_{2}$, then set $e_{2}=e_{2}^{\prime}$, "forget" that $f_{3}^{\prime}, \ldots, f_{N}^{\prime}$ were ever picked, and reset $N$ to be 2 . If this does not occur, then there is a $\beta_{2}$ in $(0,1)$ such that setting $e_{2}=\beta_{2} e_{2}^{\prime}+\sqrt{1-\beta_{2}^{2}} f_{3}^{\prime}$ and $e_{3}^{\prime}=\sqrt{1-\beta_{2}^{2}} e_{2}^{\prime}-\beta_{2} f_{3}^{\prime}$ will give $\left|\left(T e_{2}, e_{2}\right)\right|=\alpha_{2}$ and $\left|\left(T e_{3}^{\prime}, e_{3}^{\prime}\right)\right|+\sum_{i=4}^{N}\left|\left(T f_{i}^{\prime}, f_{i}^{\prime}\right)\right| \leqslant \sum_{n=3}^{N} \alpha_{n}$. Continuing in this manner yields $e_{3}, e_{4}, \ldots, e_{N}$.

Let $f_{N+1}^{\prime}$ be the element of least index in $\left\{f_{n}\right\}$ not chosen previously. If $\left|\left(T f_{N+1}^{\prime}, f_{N+1}^{\prime}\right)\right| \leqslant \alpha_{N+1}$ then put $e_{N+1}=f_{N+1}^{\prime}$. Otherwise, let $M$ be such that $\left|\left(T f_{N+1}^{\prime}, f_{N+1}^{\prime}\right)\right|<\sum_{n=N+1}^{M} \alpha_{n}$. Pick distinct vectors $f_{N+2}^{\prime}, \ldots, f_{M}^{\prime}$ from $\left\{f_{n}\right\}$ which are also distinct from all previously picked vectors and that satisfy $\left|\left(T f_{i}^{\prime}, f_{i}^{\prime}\right)\right| \leqslant$ $\left(\sum_{n=N+1}^{M} \alpha_{n}-\left|\left(T f_{N+1}^{\prime}, f_{N+1}\right)\right|\right) /(M-N)$. This procedure will then yield $e_{N+1}, \ldots, e_{M}$. Continuing in this manner will give the required basis.

(a) $\rightarrow$ (d): Fix $n$ and let $\mathcal{E}(\mathrm{n})$ be the basis obtained from $\mathcal{E}$ using Lemma 2.1 with $N(m)=2^{(m+n) !}$ and $\varepsilon(m)=1$. Let $R^{i}$ and $S^{i}$ be operators defined by

$$
\begin{aligned}
& \left(R^{i} e_{l}^{j}, e_{m}^{k}\right)= \begin{cases}\left(T e_{l}^{j}, e_{m}^{k}\right) & \text { if } i=l \text { and } i<m, \\
0 & \text { otherwise. }\end{cases} \\
& \left(S^{i} e_{l}^{j}, e_{m}^{k}\right)= \begin{cases}\left(T e_{l}^{j}, e_{m}^{k}\right) & \text { if } i<l \text { and } i=m, \\
0 & \text { otherwise. }\end{cases}
\end{aligned}
$$


Then $\left\|T_{\mathscr{E}(n)}\right\| \leqslant \Sigma\left\|R_{\mathscr{E}(n)}^{i}\right\|+\left\|S_{\mathscr{E}(n)}^{i}\right\|$, while from Lemmas 2.1 and $2.2(\mathrm{~b})$ we have $\left\|R_{\mathscr{E}(n)}^{i}\right\|,\left\|S_{\mathscr{E}(n)}^{i}\right\| \leqslant\|T\| \cdot 2^{1-(i+n) ! / 2}$. This gives $\left\|T_{\mathscr{E}(n)}\right\| \leqslant\|T\| \cdot w^{2-n ! / 2}$.

(a) $\rightarrow$ (f): We use Lemma 2.1 with $N(n)=2^{n !}$ and $\varepsilon(n)=2^{-(n+1) !}$ to find a basis $\mathcal{E}$. Let $P^{n}, R^{n}$, and $S^{n}$ be operators defined by

$$
\begin{gathered}
\left(P^{n} e_{l}^{i}, e_{m}^{j}\right)= \begin{cases}0 & \text { if } l<n \text { and } m<n, \\
\left(T e_{l}^{i}, e_{m}^{j}\right) & \text { otherwise. }\end{cases} \\
\left(R^{n} e_{l}^{i}, e_{m}^{j}\right)= \begin{cases}\left(T e_{l}^{i}, e_{m}^{j}\right) & \text { if } l=n \text { and } m<n \text { or } l<n \text { and } m=n, \\
0 & \text { otherwise. }\end{cases} \\
\left(S^{n} e_{l}^{i}, e_{m}^{j}\right)= \begin{cases}\left(T e_{l}^{i}, e_{m}^{j}\right) & \text { if } l=m=n, \\
0 & \text { otherwise. }\end{cases}
\end{gathered}
$$

For fixed $q$ and $r$, it suffices to show that $\left\|P_{\mathscr{E}}^{n}\right\|_{q r} \rightarrow 0$. Only the hardest case, $0<r<q<1$, will be shown. Let $C$ be such that $\|A+B\|_{r}<C\left(\|A\|_{r}+\|B\|_{r}\right)$. (Recall that $C=2^{1 / r-1}$ will work.) Decomposing $P^{n}$ into sums of block matrices, one has

$$
\left\|P_{\mathscr{E}}^{n}\right\|_{q r} \leqslant C\left[\left\|R_{\mathscr{E}}^{n}\right\|_{q r}+\sum_{i=1}^{\infty} C^{i+1}\left(\left\|S_{\mathscr{E}}^{n+i-1}\right\|_{q r}+\left\|R_{\mathscr{E}}^{n+i}\right\|_{q r}\right)\right] .
$$

The norm of $R_{\mathscr{E}}^{n}$ is no greater than $C$ times the sum of the norms of its two rectangular blocks. Each block has as its smallest dimension $\sum_{i=1}^{n-1} N(i)<2^{(n-1) !+1}$. The choice of $N(n)$ guarantees that no entry of $R^{n}$ is greater than $\|T\| \cdot 2^{1-n ! / 2}$. Using Lemma 2.2(b) one sees that there is a constant $\alpha$ such that

$$
\left\|R_{\mathscr{E}}^{n}\right\|_{q r}<2^{-n ! / 2+\alpha(n-1) !} \text {. }
$$

The norm of $S_{\mathscr{E}}^{n}$ can be estimated by noticing that its nonzero entries are, by Lemma 2.1(c), very nearly equal. Treating $S_{\mathscr{E}}^{n}$ as a small perturbation of a multiplier with all its nonzero entries equal, one can show that there is a constant $\beta$ such that

$$
\left\|S_{\mathscr{E}}^{n}\right\|_{q r} \leqslant 2^{n !(1 / r-1 / q-1)+\beta} .
$$

Substituting these estimates into (*), one sees that there is a constant $A$ such that

$$
\left\|P_{\mathscr{E}}^{n}\right\|_{q r} \leqslant A 2^{-n ! \min (1 / 2,1+1 / q-1 / r)+\alpha(n-1) !} \text {. }
$$

As long as $1+1 / q-1 / r>0,\left\|P_{\mathscr{E}}^{n}\right\|_{q r} \rightarrow 0$ as $n \rightarrow \infty$.

Notice that Theorem 2.3(e) is a significant improvement upon Theorem 1.2(e). Considering the importance of Kuroda's theorem in the proof of Theorem 1.2(e), Theorem 2.3(e) seems to suggest that Kuroda's result can be refined. A natural conjecture is: let $\mathscr{G}$ be any convex ideal strictly containing $\mathcal{C}_{1}$. (See Stout [16] for definitions. Convex ideals have also been called interpolating ideals and majorizing ideals, e.g. in Russu [14].) Then for any selfadjoint operator $A$ there is a diagonal operator $D$ such that $A-D \in G$. Further, if $G$ is symmetrically normed and $\varepsilon>0$ is given, then $D$ can be chosen so that $A-D$ has 9 -norm less than $\varepsilon$.

Let $\sigma_{\mathscr{E}}(T)$ denote the spectrum of $T$ in $\mathscr{B}_{\mathscr{G}}$.

2.4. Corollary. For any $T$ in $\mathscr{B}(\mathcal{H})$ there is a basis $\mathcal{E}$ such that $\sigma_{\mathfrak{E}}(T)$ has at most two accumultion points. 
Proof. Let $\lambda \in W_{e}(T)$. Then $0 \in W_{e}(T-\lambda)$ so, by Theorem 2.3(e), there is a basis $\mathcal{E}$ such that 0 is the only accumultion point of $\sigma_{\mathscr{E}}(T-\lambda)$. Then 0 and $\lambda$ are the only accumulation points of $\sigma_{\mathscr{E}}(T)$.

2.5. Corollary. If $T \in \mathscr{B}(\mathcal{H})$ then

(a) $T_{\mathscr{E}} \in \mathscr{K}(\Re(\mathscr{H}))$ for all bases $\mathcal{E}$ iff $T$ is compact,

(b) $T_{\mathscr{E}} \in \mathcal{K}\left(\mathscr{B}(\mathcal{H})\right.$ ) for some basis $\mathcal{E}$ iff $0 \in W_{e}(T)$.

Proof. To prove (b) it is sufficient to show that if $0 \notin W_{e}(T)$, then for any basis $\mathcal{E}=\left\{e_{n}\right\}, T_{\mathscr{E}} \notin \mathscr{K}(\mathscr{B}(\mathcal{H}))$. By Theorem 1.2(b) there is a constant $d>0$ such that $\left|\left(T e_{n}, e_{n}\right)\right| \geqslant d$ for all but finitely many $n$. Thus $T_{\mathscr{E}}\left(e_{n} \otimes e_{n}\right)$ is not converging in norm as $n \rightarrow \infty$, so $T_{\mathscr{E}}$ is not compact.

Both directions of (a) need to be proven. If $T$ is not compact then there is a $\lambda$ in $W_{e}(T)$ with $\lambda \neq 0$. By Theorem 1.2 there is a basis $\mathcal{E}=\left\{e_{n}\right\}$ such that $\left(T e_{n}, e_{n}\right) \rightarrow$ $\lambda$. As was shown previously, $T_{\mathscr{E}}$ is not compact. Finally, assume $T$ is compact and let $\mathcal{E}=\left\{e_{n}\right\}$ be any basis. Define $P_{n}$ to be the projection onto the first $n$ elements of $\mathcal{E}$. Since $P_{n} T P_{n} \rightarrow T$ in norm, $\left(P_{n} T P_{n}\right)_{\mathscr{E}} \rightarrow T_{\mathscr{E}}$. Since $\left(P_{n} T P_{n}\right)_{\mathscr{E}}$ is of finite rank in $\mathscr{B}(\mathscr{B}(\mathcal{H})), T_{\mathscr{E}}$ is compact.

One consequence of Theorem 2.3 is that if there is a basis $\mathcal{E}$ such that $T \in \operatorname{kernel}\left(\right.$ hull $\left.\left(\mathcal{K}_{\mathscr{E}}\right)\right)$, then there is a basis $\mathscr{F}$ such that $T_{\mathscr{f}}(T)$ is compact. In Stout [17] it is further shown that $T_{\mathscr{E}}\left(T_{\S}(T)\right)$ is compact, though it may occur that $T_{\mathscr{\delta}}(T)$ is not.

3. Further remarks. One can prove the following theorem by a more careful use of Lemmas 2.1 and 2.2. However, the details are uninstructive and will not be given. In part (c) we use a generalized Schatten class, which we have defined by approximation numbers. In Pietsch [10] there is an axiomatic theory of $s$-numbers, any of which could be used to define Schatten classes, where all definitions agree on Hilbert space. Approximation numbers are the largest possible $s$-numbers [10, Theoem 3.2], which implies that our Schatten classes are the smallest possible.

3.1. THEOREM. For $T$ in $\mathscr{B}(\mathcal{H})$ the following are equivalent.

(a) $0 \in W_{e}(T)$.

(b) $T=0$, or there is a basis $\left\{e_{n}\right\}$ such that the doubly indexed sequence $\left(T e_{n}, e_{m}\right)$ is in $\bigcap_{p>2} l_{p} \backslash \cup_{q<2} l_{q}$.

(c) There is a basis $\mathcal{E}$ such that for all $q$ in $(0, \infty], r$ in $(q /(q+1), \infty]$, and $p$ in $(2 /(1+\min (0,1 / q-1 / r)), \infty], T_{\mathcal{E}} \in \mathcal{C}_{p}\left(\mathcal{C}_{q}, \mathcal{C}_{r}\right)$.

Related to Theorems 3.1(b) and 2.3(e) there is the following open question: given an operator $T$ such that $0 \in W_{e}(T)$, and a sequence $\left\{\alpha_{n}\right\}$ which is not in $l_{2}$, does there exist a basis $\left\{e_{n}\right\}$ and a bijection $\pi$ from $N \times N$ onto $N$ such that $\left|\left(T e_{n}, e_{n}\right)\right|$ $<\alpha_{\pi(n, m)}$ for all $n$ and $m$ ? This question is similar to two better known ones also concerned with matrix representations.

(i) Given a normal operator $N$, does there exist a diagonal operator $D$ such that $N-D \in C_{2}$ ?

(ii) Given an arbitrary operator $T$, does there exist a basis $\left\{e_{n}\right\}$ and bounded linear operator $S$ such that $\left|\left(T e_{n}, e_{m}\right)\right|=\left(S e_{n}, e_{m}\right)$ ? 
Question (i) is in the Weyl-von Neumann-Kuroda-Berg sequence of results and was recently answered in the affirmative by Voiculescu [21]. Question (ii) has been mentioned by Halmos and is related to work of Sunder [19].

The following proposition shows that the restriction $r>q /(q+1)$ in Theorems 2.3(f) and 3.1(c) is necessary.

3.2. Proposition. Let $P$ be an orthogonal projection with infinite-dimensional range. If $q \in(0, \infty]$ and $r=q /(q+1)$ then $P_{\mathcal{E}} \notin \Re\left(\mathcal{C}_{q}, \mathcal{C}_{r}\right)$ for any basis $\mathcal{E}$.

Proof. $P$ is a positive operator with infinite trace, and hence $\Sigma\left(P e_{n}, e_{n}\right)=+\infty$ for any basis $\mathcal{E}=\left\{e_{n}\right\}$. Therefore, for any $q$, there is a sequence $\left\{a_{n}\right\}$ such that $\left\{a_{n}\right\} \in l_{q}$ but $\left\{\left(P e_{n}, e_{n}\right) a_{n}\right\} \notin l_{r}$, where $r=q /(q+1)$. If $A$ is a diagonal matrix with diagonal $\left\{a_{n}\right\}$, then $A \in \mathcal{C}_{q}$ and $P * A \notin \mathcal{C}_{r}$.

This raises the following question: suppose $T \in \mathscr{B}(\mathcal{H})$ and there is a basis $\left\{e_{n}\right\}$ such that $\Sigma_{n}\left|\left(T e_{n}, e_{n}\right)\right|<\infty$. Does there exist a basis $\mathscr{F}$ such that $T_{\mathscr{F}} \in$ $\mathscr{B}\left(\mathscr{B}(\mathcal{H}), \mathcal{C}_{1}\right)$ ? Use of duality shows that if $T \in \mathcal{C}_{1}$ then $T_{\mathscr{E}} \in \mathscr{B}\left(\mathscr{B}(\mathcal{H}), \mathcal{C}_{1}\right)$ for all bases $\mathcal{E}$. (This fact was used in Johnson and Williams [6.) Further, this is a characterization of trace class operators.

It is also possible to show that the restrictions on $p$ in Theorem 3.1(c) cannot be relaxed. However, one can improve the theorem by adding that if $T$ is not 0 then $T_{\mathscr{E}}$ can also be made to be 1-1 and have dense range. (It can never be onto.) To do this it is necessary and sufficient to show that $\mathcal{E}$ can be chosen so that all entries of the matrix of $T$ are nonzero. This can be accomplished by incorporating the techniques of Radjavi and Rosenthal [12].

\section{REFERENCES}

1. J. Anderson, Derivations, commutators and the essential numerical range, $\mathrm{Ph} . \mathrm{D}$. Thesis, Indiana University, 1971.

2. J. Anderson and J. G. Stampfli, Compressions and commutators, Israel J. Math. 10 (1971), 433-441.

3. G. Bennett, Unconditional convergence and almost everywhere convergence, $Z$. Wahrscheinlichkeitstheorie und Verw. Gebiete 34 (1976), 135-155.

4. __ Schur multipliers, Duke Math J. 44 (1977), 603-639.

5. F. F. Bonsall and J. Duncan, Numerical ranges of operators on normed spaces and of elements of normed algebras, Cambridge Univ. Press, London, 1971.

6. B. E. Johnson and J. P. Williams, The range of a normal derivation, Pacific J. Math 58 (1975), $105-122$.

7. S. T. Kuroda, On a theorem of Weyl-von Neumann, Proc Japan Acad. 34 (1958), 11-15.

8. S. Kwapien and A. Pelczynski, The main triangle projection in matrix spaces and its applications, Studia Math. 38 (1970), 43-68.

9. C. A. McCarthy, $e_{p}$, Israel J. Math 5 (1967), 249-271.

10. A. Pietsch, s-numbers of operators in Banach spaces, Studia Math. 51 (1974), 201-223.

11. C. Pommerenke, Univalent functions, Hubert, Göttingen, 1975.

12. H. Radjavi and P. Rosenthal, Matrices for operators and generation of $B(\mathcal{B C})$, J. London Math. Soc. (2) 2 (1970), 557-560.

13. W. H. Ruckle, Hadamard multipliers of infinite matrices, Indiana Univ. Math. J. 24 (1975), 949-957.

14. G. I. Russu, On intermediate symmetrically normed ideals, Functional Anal. Appl. 3 (1969), $163-165$.

15. L. Schur, Bemerkungen zur Theorie der beschrankten Bilinearformen mit unendlich vielen Veranderlichen, J. Reine Angew. Math. 140 (1911), 1-28.

16. Q. F. Stout, Convex ideals of $\mathscr{B}(\mathcal{H})$ (to appear). 
17. . Schur multiplication on $\mathscr{B}\left(l_{p}, l_{q}\right)$ (to appear).

18. G. P. H. Styan, Hadamard products and multivariate statistical analysis, Linear Algebra and Appl. 6 (1973), 217-240.

19. V. S. Sunder, Absolutely bounded matrices, Indiana Univ. Math. J. 27 (1978), 919-927.

20. N. Th. Varopoulos, $A$ theoem on operator algebras, Math. Scand. 37 (1975), 173-182.

21. D. Voiculescu, Some results on norm ideal perturbations of Hilbert space operators, Rev. Roumaine Math. Pures Appl. (to appear).

Department of Mathematical Sciences, State University of New York, Center at Binghamton, Binghamton, New York 13901 